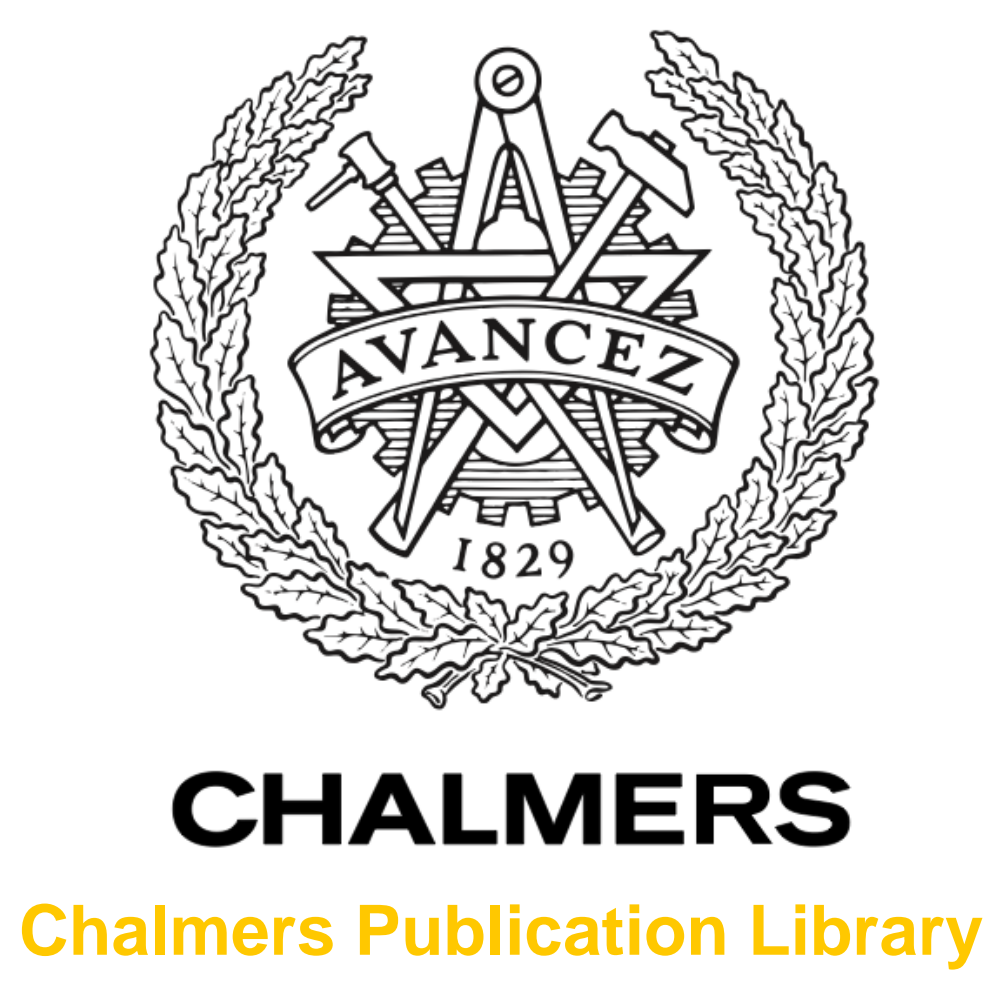

\title{
Validating the Harmonic Balance Method for Turbomachinery Tonal Noise Predictions
}

This document has been downloaded from Chalmers Publication Library $(\mathrm{CPL})$. It is the author's version of a work that was accepted for publication in:

Proceedings of the 55th AIAA Aerospace Sciences Meeting

\author{
Citation for the published paper: \\ Lindblad, D. ; Andersson, N. (2017) "Validating the Harmonic Balance Method for \\ Turbomachinery Tonal Noise Predictions". Proceedings of the 55th AIAA Aerospace \\ Sciences Meeting(AIAA 2017-1171),
}

http://dx.doi.org/10.2514/6.2017-1171

Downloaded from: http://publications.lib.chalmers.se/publication/247699

Notice: Changes introduced as a result of publishing processes such as copy-editing and formatting may not be reflected in this document. For a definitive version of this work, please refer to the published source. Please note that access to the published version might require a subscription. 
55th AIAA Aerospace Sciences Meeting, AIAA SciTech Forum, 9 - 13 January, Grapevine, Texas

\title{
Validating the Harmonic Balance Method for Turbomachinery Tonal Noise Predictions
}

\author{
Daniel Lindblad*, and Niklas Andersson ${ }^{\dagger}$ \\ Chalmers University of Technology, Gothenburg, SE-412 96, Sweden
}

\begin{abstract}
A computational method for predicting turbomachinery tonal noise is presented. It is based on the Harmonic Balance method, which solves for the dominant frequencies of the flow generated by bladerow interaction. Coupling between the resolved frequencies is furthermore enabled since the Harmonic Balance method is applicable to the nonlinear Euler or Navier-Stokes equations. The implementation is also validated against two cases from the Fourth Computational Aeroacoustics Workshop on Benchmark Problems hosted by NASA. The computational setup is explained in detail and specific challenges encountered in both benchmark problems are discussed. Results generally show very good agreement with data published by several other authors, giving confidence in the capability of the method. Some remaining challenges for the implemented method necessary to compute full scale turbomachinery noise are also identified and discussed.
\end{abstract}

\section{Nomenclature}

\begin{tabular}{ll} 
Roman & \\
$a$ & Speed of sound, $\mathrm{m} / \mathrm{s}$ \\
$c$ & Axial chord, m \\
$D$ & Time Spectral Derivative matrix, $1 / \mathrm{s}$ \\
$\mathcal{F}_{j}$ & Convective flux vector in $j$ direction \\
$G$ & Damping matrix \\
$i$ & Imaginary unit \\
$k$ & Reduced frequency \\
$n$ & Harmonic number \\
$N$ & Number of blades \\
$N_{h}$ & Number of harmonics \\
$N_{t}$ & Number of time levels \\
$p$ & Pressure, Pa \\
$\mathcal{Q}$ & State vector \\
$\hat{\mathcal{Q}}_{n}$ & Fourier coefficient vector for mode $n$ \\
$r$ & Radial coordinate, $\mathrm{m}$ \\
$R$ & Specific gas constant for air, $\mathrm{J} /(\mathrm{kg} \mathrm{K})$ \\
$\mathcal{R}$ & Residual flux vector \\
$S$ & Phase shift matrix \\
$t$ & Time, s \\
$T$ & Time period, s \\
$u, U$ & Velocity, m/s \\
$V$ & Volume of computational cell, $\mathrm{m}^{3}$ \\
$x$ & Axial coordinate, $\mathrm{m}$ \\
$x_{j}$ & Cartesian coordinate, $\mathrm{m}$ \\
\hline
\end{tabular}

${ }^{*}$ Ph.D. Student, Department of Applied Mechanics, Chalmers University of Technology, Gothenburg, Sweden

${ }^{\dagger}$ Assistant Professor, Department of Applied Mechanics, Chalmers University of Technology, Gothenburg, Sweden

Copyright (C) 2017 by the American Institute of Aeronautics and Astronautics, Inc. The U.S. Government has a royalty-free license to exercise all rights under the copyright claimed herein for Governmental purposes. All other rights are reserved by the copyright owner. 


$\begin{array}{ll}\text { Greek } & \\ \alpha & \text { Inflow angle } \\ \beta & \text { Phase shift, rad } \\ \gamma & \text { Ratio of specific heats } \\ \delta_{i, j} & \text { Kronecker delta } \\ \epsilon & \text { Velocity perturbation amplitude } \\ \zeta & \text { Direction of phase shift } \\ \theta & \text { Angle, rad } \\ \theta_{S} & \text { Angle spanned by two adjacent blades, rad } \\ \rho & \text { Density, kg/m }{ }^{3} \\ \tau & \text { Pseudo time, s } \\ \omega & \text { Angular frequency, rad/s } \\ \Omega & \text { Rotational speed, rad/s } \\ \text { Subscript } & \\ 0 & \text { Stagnation property } \\ i & \text { Computational cell } \\ l, m & \text { Time level } \\ \text { Abbreviations } & \\ \text { CAA } & \text { Computational Aeroacoustics } \\ \text { CFD } & \text { Computational Fluid Dynamics } \\ \text { CFL } & \text { Courant Friedrichs Lewy } \\ \text { CROR } & \text { Counter Rotating Open Rotor } \\ \text { NLFD } & \text { Non-Linear Frequency Domain Method } \\ \text { NLH } & \text { Non-Linear Harmonic Method } \\ \text { OGV } & \text { Outlet Guide Vane } \\ \text { SPL } & \text { Sound Pressure Level } \\ \text { (U)RANS } & \text { (Unsteady) Reynolds Averaged Navier Stokes }\end{array}$

\section{Introduction}

Transient flows within the turbomachinery components of an aircraft engine generate fluctuating pressure fields that to some extent will radiate into the surroundings and be perceived as noise. In present-day turbofan engines a large part of the turbomachinery noise stems from the fan stage in the bypass duct. This noise is furthermore an important contributor to the overall noise levels of the engine. ${ }^{1}$ In the future, the Counter Rotating Open Rotor (CROR) engine may become an alternative to turbofans since it can achieve a higher propulsive efficiency by trading the nacelle for larger fan blades. A negative consequence of this is that noise radiation from the fan blades also will increase. In addition, it was pointed out by Peake et al. ${ }^{1}$ that in contrast to turbofans, it is very difficult to limit the radiation efficiency of noise from a CROR by careful selection of blade counts. Quiet and efficient fan blades must thus be developed to enable the fuel efficient CROR architecture to meet the strict noise regulations necessary to be realized in the future. A fundamental understanding of the noise generation mechanisms combined with accurate noise prediction tools are vital to achieve this task.

Turbomachinery noise contains both tonal and broadband components. Tonal noise is emitted at discrete frequencies and constitute of three main sources. The first two are linked to volume displacement and steady loading of the passing blades and can thus be predicted by steady state methods. ${ }^{2}$ The last one is linked to transient loading of the blades resulting from their interaction with wakes and potential fields bound to adjacent bladerows. Broadband noise is on the other hand generated across a large frequency spectrum and is linked to turbulence. A more extensive review of the different noise sources found in a turbofan is given by Envia et al. ${ }^{3}$ and for both turbofan and CRORs by Peake et al. ${ }^{1}$

A range of numerical methods that enables different levels of fidelity at different computational costs are currently available to aid designers in predicting these noise sources. They range from fast analytical methods to computer intensive methods based on Computational Fluid Dynamics (CFD). The latter alternative have the capability of resolving both tonal and broadband noise sources, if executed in time accurate mode on 
high resolution grids. This makes high resolution CFD generic in nature but also too computationally expensive for design purposes. If the broadband noise sources are neglected the computational cost of CFD can be reduced by solving for the Unsteady Reynolds Averaged Navier Stokes (URANS) equations instead. However, for a CROR engine the broadband noise may be an important contributor to the overall noise levels, ${ }^{4}$ which must be appreciated when this assumption is used. From a design perspective the tonal noise sources are nevertheless relevant since they are deterministic in nature. This makes them suitable for noise control using for example acoustic liners and tailored blades.

Tonal noise generated by the interaction between wakes and a downstream bladerow can in many cases be accurately predicted by linearized methods. These are based on solving for linear perturbations on top of a non-linear steady mean flow and are well established for fan noise. They have also been successfully applied to Open Rotors by for example Sharma et al. ${ }^{2}$ A disadvantage of the method is that non-linearities within the flow, including coupling between acoustic modes, cannot be accounted for. In addition, a fully coupled rotor-stator or rotor-rotor computation appears very cumbersome with this approach, ${ }^{3}$ implying that all interaction noise sources cannot be included in the analysis. Non-linear effects can be accounted for by solving for the URANS equations using both time accurate and frequency based approaches. Time accurate simulations of turbomachinery flows generally requires the whole annulus to be included in the computational domain. This is because periodicity at periodic boundaries can not directly be achieved if the blade counts does not match. One solution to this problem is the Chorochronic method of Gerolymos et al., ${ }^{5}$ which enables the computational domain to be reduced to one blade per row. This method has successfully been applied to both turbofan ${ }^{6}$ and $\mathrm{CROR}^{7}$ noise. Frequency based approaches target computational efficiency by solving directly for the deterministic frequencies of the flow and also enable computational domains with only one blade per row. An early approach was the Non-Linear Harmonics (NLH) method proposed by He et al. ${ }^{8}$ In this method, harmonic disturbances around a steady mean field are solved for in the frequency domain. These are then introduced back into the mean flow equations as deterministic stresses similar to the Reynolds stresses arising from the Reynolds decomposition. A similar approach is the classical Harmonic Balance method in which an harmonic form of the solution variables are assumed and substituted into the governing equations. A set of coupled equations for the harmonic amplitudes are then obtained by requiring that the original equation is satisfied for each individual frequency component. ${ }^{9}$ Hall et al. ${ }^{10}$ later showed that the balancing procedure could be removed if the problem was recast in the time domain. This method will be referred to as the Harmonic Balance method for the remainder of this paper, other names include the Time-Spectral or High Dimensional Harmonic Balance method. It is also worth noting that the Harmonic Balance method can be formulated in the frequency domain, known as the Non-Linear Frequency Domain (NLFD) method. ${ }^{11}$ Application of frequency domain methods to the non-linear URANS equations for noise predictions have been done by several authors,.$^{6,12-15}$

To the authors' knowledge there is however still very little work available in the literature on validating the Harmonic Balance method for turbomachinery aeroacoustics. This paper aims to expand this knowledge by presenting results obtained with the Harmonic Balance solver recently implemented into Chalmers' in-house CFD/CAA tool G3D::Flow. In section II the Harmonic Balance equations are first derived. An in-depth description of the computational setup used to validate the implementation is also presented. Two relevant benchmark problems from the Fourth Computational Aeroacoustics Workshop on Benchmark Problems hosted by NASA are considered in this work. They are known as the Sigle Airfoil Gust Response Problem ${ }^{16}$

and the Cascade Gust Interaction Problem. ${ }^{17}$ Results from the G3D::Flow code are compared to data published on the same problems by several authors in section III. It is found that the Harmonic Balance method generally compares well with these results throughout all cases.

\section{Computational Method}

\section{II.A. Flow solver}

The G3D::Flow code can be used for inviscid and viscous analysis of both compressible and incompressible flows using a variety of URANS, DES and LES turbulence models. In this work the compressible, inviscid Euler equations are considered

$$
\frac{\partial \mathcal{Q}}{\partial t}+\frac{\partial \mathcal{F}_{j}}{\partial x_{j}}=0
$$


Here, $\mathcal{Q}$ is the state vector containing the conservative variables and $\mathcal{F}_{j}$ contains pressure and convective fluxes. The gas is assumed to be calorically perfect and obey the perfect gas equation of state with the thermodynamic properties of air. The equations are discretized on a structured, multi-block grid using the finite volume method. A low dissipation, third-order accurate, upwind scheme based on wave splitting is used to reconstruct convective fluxes on cell faces and time integration is done using a standard three-stage, second order accurate, Runge-Kutta cycle.

\section{II.B. Harmonic Balance Technique}

To derive the Harmonic Balance equations it is assumed that the conservative variables present in the state vector $\mathcal{Q}$ are periodic in time with a known period $T$. This assumption implies that the solution can be expressed as a Fourier series in time, with spatially varying coefficients

$$
\mathcal{Q}\left(t, x_{j}\right)=\sum_{n=-\infty}^{\infty} \hat{\mathcal{Q}}_{n}\left(x_{j}\right) e^{i \omega_{n} t}
$$

Here, $\omega_{n}=2 \pi n / T$ is the angular frequency of the $n^{t h}$ harmonic. The above expression is approximated by assuming that the flow can be accurately described by a limited number of harmonics

$$
\mathcal{Q}(t) \approx \sum_{n=-N_{h}}^{N_{h}} \hat{\mathcal{Q}}_{n} e^{i \omega_{n} t}
$$

Note that the spatial dependency was omitted here for brevity. The time period is now divided into $N_{t}=$ $2 N_{h}+1$ equally spaced points and a new state vector $\mathcal{Q}^{*}$ is introduced. $\mathcal{Q}^{*}$ contains the solution at each time level $t_{l}=l T / N_{t}$ according to

$$
\mathcal{Q}^{*}=\left[\mathcal{Q}_{0}, \mathcal{Q}_{1}, \ldots, \mathcal{Q}_{N_{t}-1}\right]^{T}
$$

The new state vector allows the Fourier coefficients in Eq. (3) to be obtained via a discrete Fourier transform over all time levels as

$$
\hat{\mathcal{Q}}_{n}=\frac{1}{N_{t}} \sum_{l=0}^{N_{t}-1} \mathcal{Q}_{l} e^{-i \omega_{n} t_{l}}
$$

By differentiating Eq. (3) with respect to time and combining the resulting expression with Eq. (5), an approximation of the time derivative at time level $m$ is obtained according to

$$
\frac{\partial \mathcal{Q}_{m}}{\partial t} \approx \sum_{n=-N_{h}}^{N_{h}} i \omega_{n}\left(\frac{1}{N_{t}} \sum_{l=0}^{N_{t}-1} \mathcal{Q}_{l} e^{-i \omega_{n} t_{l}}\right) e^{i \omega_{n} t_{m}}
$$

Interchanging the order or summation and combining the exponential terms further yields

$$
\frac{\partial \mathcal{Q}_{m}}{\partial t} \approx \sum_{l=0}^{N_{t}-1}\left(\frac{i}{N_{t}} \sum_{n=-N_{h}}^{N_{h}} \omega_{n} e^{i \omega_{n}\left(t_{m}-t_{l}\right)}\right) \mathcal{Q}_{l}
$$

This equation represents a high order finite difference approximation of the time derivative, with a stencil spanning over all time levels. In matrix notation this may be expressed as

$$
\frac{\partial \mathcal{Q}^{*}}{\partial t} \approx D \mathcal{Q}^{*}
$$

$D$ is known as the time spectral derivative matrix and is block structured with $N_{t} \times N_{t}$ blocks. Each block contains a diagonal matrix of size $N_{v a r} \times N_{v a r}$, where $N_{v a r}$ are the number of conservative variables present in $\mathcal{Q}$. The entries of the diagonal matrix in block $(m, l)$ are equal and will here be referred to as $d_{m, l}$. They can be identified to be given by the expression within the parenthesis of Eq. (7). This expression may be simplified by evaluating the summation, for a complete derivation see Ref. 18 


$$
d_{m, l}= \begin{cases}0, & l=m \\ \frac{\pi}{T}(-1)^{(m-l)} \csc \left(\frac{\pi(m-l)}{N_{t}}\right), & l \neq m\end{cases}
$$

A governing equation for the new state vector may now be formulated in which the time derivative is replaced by Eq. (8)

$$
D \mathcal{Q}^{*}+\frac{\partial \mathcal{F}_{j}^{*}}{\partial x_{j}}=0
$$

This represents a coupled set of mathematically steady state equations for the periodic solution at each time level. The new flux vector furthermore contains the flux at each time level

$$
\mathcal{F}_{j}^{*}=\left[\mathcal{F}_{j, 0}, \mathcal{F}_{j, 1}, \ldots, \mathcal{F}_{j, N_{t}-1}\right]^{T}
$$

Eq. (10) is discretized in space and a pseudo time derivative is introduced to drive the resulting system of equations towards steady state using local time stepping

$$
V_{i} \frac{\partial \mathcal{Q}_{i}^{*}}{\partial \tau}+V_{i} D \mathcal{Q}_{i}^{*}+\mathcal{R}^{*}=0
$$

Here, index $i$ represents a specific cell in the computational mesh. The residual vector contains the discretized fluxes and can be computed independently for each time level

$$
\mathcal{R}^{*}=\left[\mathcal{R}\left(\mathcal{Q}_{0}\right), \mathcal{R}\left(\mathcal{Q}_{1}\right), \ldots, \mathcal{R}\left(\mathcal{Q}_{N_{t}-1}\right)\right]^{T}
$$

In each stage of the Runge-Kutta cycle the Harmonic Balance solver starts by looping through all time levels to compute the residual vector $\mathcal{R}^{*}$. Instead of adding the time spectral derivative directly to the residual at each time level before updating the state vector $\mathcal{Q}^{*}$, the time spectral derivative is treated implicitly within the Runge-Kutta cycle according to the method proposed by Campobasso et al. ${ }^{19}$

\section{II.C. Phase-Shifted Periodic Boundary Condition}

In the second benchmark problem a two dimensional cascade representing an unrolled section of a turbofan outlet guide vane (OGV) is considered. At the inlet, wakes from an upstream fan are prescribed with an tangential periodicity different from the spacing of the OGVs. This requires the use of phase shifted periodic boundary conditions if the computational domain is to be reduced to include only one blade. A general derivation of the boundary condition for Harmonic Balance solvers is presented here in polar coordinates. The derivation is however equally valid for a two dimensional cascade if the coordinate transformation $y=r \theta$ is done. To begin with, it is observed that the flow in an adjacent blade passage is equal to the flow in the simulated blade passage at a shifted time $t+\Delta t$. This may be expressed as

$$
\mathcal{Q}\left(x, r, \theta+\zeta \theta_{S}, t\right)=\mathcal{Q}(x, r, \theta, t+\Delta t)
$$

Here, $\theta_{S}=2 \pi / N_{2}>0$ is the angle spanned by two adjacent blades and $\zeta= \pm 1$ represent the direction in which the phase shift is performed. If the only source of unsteadiness in the blade passage is due to the periodic passing of an adjacent blade row, the time shift can be computed as $\Delta t=\zeta \beta / \omega$, where $\omega=2 \pi / T$ is the fundamental frequency of the flow and $\beta$ the phase shift ${ }^{5}$

$$
\beta=-2 \pi \operatorname{sign}\left(\Omega_{1}-\Omega_{2}\right) \frac{N_{1}-N_{2}}{N_{2}}
$$

In this equation, $\Omega$ and $N$ represent the rotational speed and blade count of the current (1) and adjacent (2) blade row respectively. By substituting Eq. (3) into Eq. (14) one obtains

$$
\sum_{n=-N_{h}}^{N_{h}} \hat{\mathcal{Q}}_{n}\left(x, r, \theta+\zeta \theta_{S}\right) e^{i \omega_{n} t}=\sum_{n=-N_{h}}^{N_{h}} \hat{\mathcal{Q}}_{n}(x, r, \theta) e^{i \omega_{n}(t+\Delta t)}
$$

Requiring that each pair of elements in the two series is equal and inserting the expression for $\Delta t$ presented above yields 


$$
\hat{\mathcal{Q}}_{n}\left(x, r, \theta+\zeta \theta_{S}\right)=\hat{\mathcal{Q}}_{n}(x, r, \theta) e^{i n \zeta \beta}
$$

The solution in an adjacent blade passage thus can be obtained by multiplying each Fourier coefficient by a complex exponential. Equation (17) is now inserted into Eq. (3) and the resulting expression is evaluated at time level $m$

$$
\mathcal{Q}_{m}\left(x, r, \theta+\zeta \theta_{S}\right)=\sum_{n=-N_{h}}^{N_{h}}\left[\left(\frac{1}{N_{t}} \sum_{l=0}^{N_{t}-1} \mathcal{Q}_{l}(x, r, \theta) e^{-i \omega_{n} t_{l}}\right) e^{i n \zeta \beta}\right] e^{i \omega_{n} t_{m}}
$$

By rearranging the order of summation, a matrix relation between the new state vector and its phase shifted counterpart $\mathcal{Q}^{*}\left(\theta+\zeta \theta_{S}\right)$ is obtained

$$
\mathcal{Q}^{*}\left(\theta+\zeta \theta_{S}\right)=S \mathcal{Q}^{*}(\theta)
$$

$S$ has the same structure as $D$, which was described in the previous section. The elements of each diagonal matrix can be shown to be ${ }^{20}$

$$
s_{m, l}=\frac{1}{N_{t}}\left(1+2 \sum_{n=1}^{N_{h}} \cos \left(\left[\frac{2 \pi(m-l)}{N_{t}}+\zeta \beta\right] n\right)\right)
$$

If the number of blades in each blade row match, $\beta=0$ according to Eq. (15). In this special case, it is found that Eq. (20) yields the identity matrix, and thus a normal periodic boundary condition is obtained. The phase shifted boundary condition is applied at both periodic boundaries using a two layer ghost cell technique. The ghost cell values are set by phase shifting the values at the opposite side of the interface, after which the normal flux routines are applied for the boundary faces. It should also be emphasized that this type of boundary condition allows another coupling between the time levels in addition to the time spectral derivative, as can be seen in Eq. (19).

\section{II.D. Computational Setup}

\section{II.D.1. Single Airfoil Gust Response}

The first benchmark problem was defined by Scott in Ref. 16. It has been designed to validate Computational Aeroacoustics (CAA) codes by computing the noise generated from the impingement of velocity gusts onto an isolated airfoil. Several cases were investigated in this work, in which both a loaded and unloaded airfoil were subjected to vortical velocity gusts of different frequencies and wavelengths. In this way the problem isolates some of the noise generation mechanisms found in a turbomachine when wakes from an upstream bladerow impinges on a rotor or stator vane. A schematic view of the computational domain is presented in figure 1.

A structured grid with a C-H-C topology was used to enable a high mesh density near the airfoil in combination with a good far field resolution at a reasonable cell count. The grid size was set to achieve a minimum of 23 points per gust wavelength in each case studied, with a substantially higher resolution near the airfoil. In addition, a buffer zone was placed ahead of the outlet in which the grid was stretched in the axial direction to a final cell length of $1 c$, where $c$ denotes axial chord. The airfoil geometries were given in Ref. 16 and represents an unloaded Joukowski airfoil at zero degrees angle of attack and a cambered airfoil at $2^{\circ}$ angle of attack. All grids were generated with the in-house meshing software G3DMesh and an example is presented in figure 2 . 


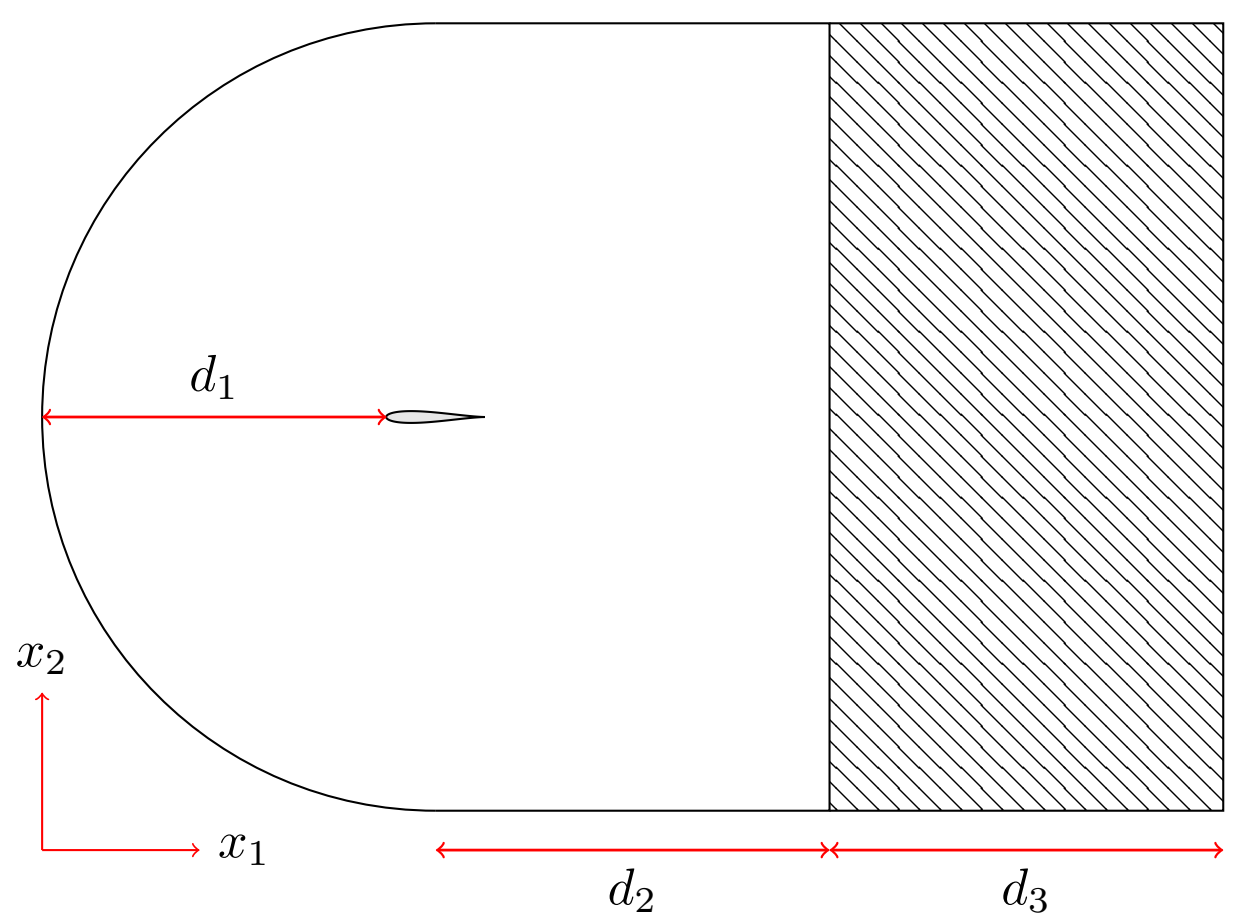

Figure 1: Computational domain for Single Airfoil Gust Response problem.

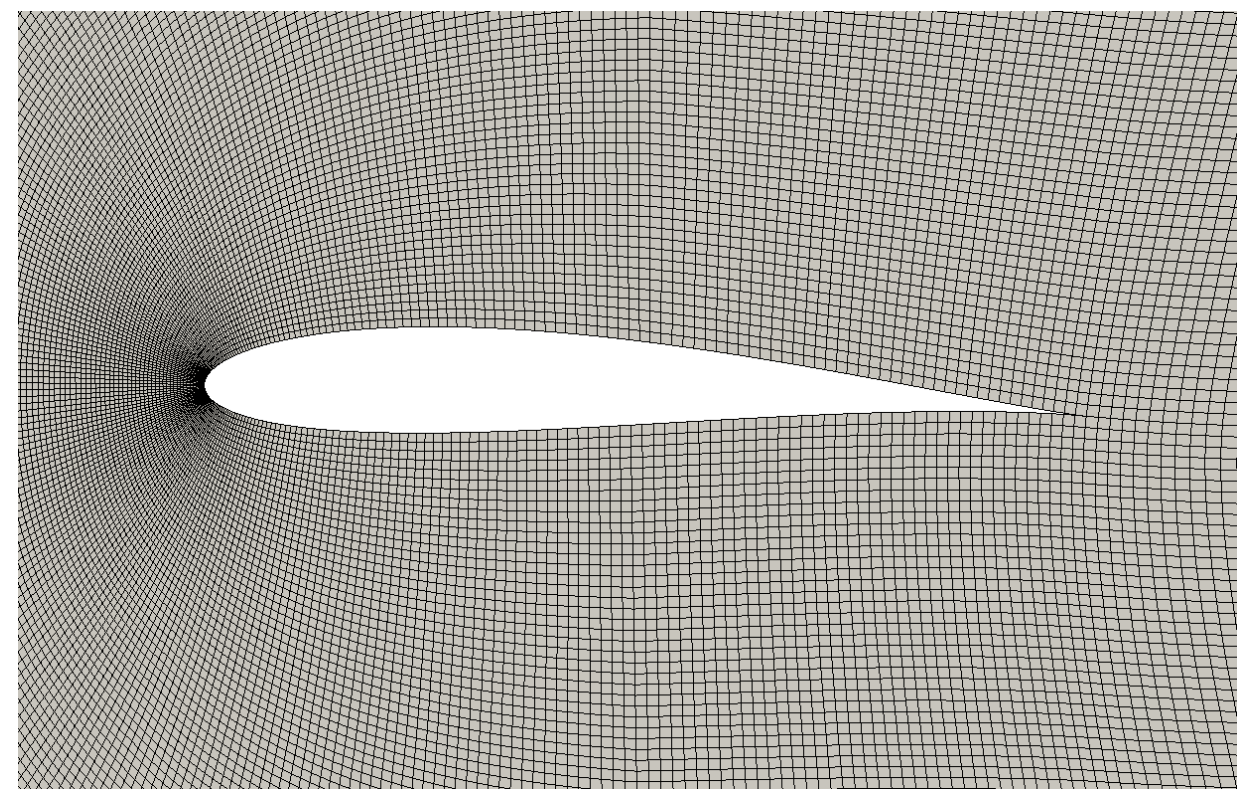

Figure 2: Computational mesh for cambered Joukowski airfoil. 
Free-slip boundary conditions were specified along the airfoil surface and no explicit enforcement of the Kutta condition was applied at the trailing edge. A one-dimensional absorbing boundary condition was furthermore used at all free-stream boundaries to specify a steady mean flow of $U_{\infty}=1.0 \mathrm{~m} / \mathrm{s}, \rho_{\infty}=\gamma=$ $1.4 \mathrm{~kg} / \mathrm{m}^{3}$, and $p_{\infty}=4 \mathrm{~Pa}$, corresponding to a Mach number of $\mathrm{Ma}_{\infty}=0.5$. The one dimensional formulation was sufficient for this case since the acoustic waves generated by the interaction travels almost radially out from the airfoil and thus exit almost normal to the boundary. Reflections from the outlet were furthermore prevented by the buffer zone. A vortical velocity gust containing a single harmonic was also added on top of the freestream conditions along all boundaries not connected to the buffer zone ${ }^{16}$

$$
\begin{aligned}
\mathbf{u}^{\prime} & =\epsilon U_{\infty} \mathbf{a} \cos (\mathbf{k} \cdot \mathbf{x}-\omega t) \\
\mathbf{a} & =\frac{\sqrt{2}}{2}\left[\begin{array}{c}
-1 \\
1
\end{array}\right] \\
\mathbf{k} & =\left[\begin{array}{l}
k_{1} \\
k_{2}
\end{array}\right]
\end{aligned}
$$

The gust amplitude and wave numbers were set to $\epsilon=0.02$ and $k_{1}=k_{2}=(2 / c) k$ respectively, where $k$ is the reduced frequency based on half the airfoil chord

$$
k=\frac{\omega c}{2 U_{\infty}}
$$

Two values of $k$ were investigated in this work, namely $k=1.0$ and $k=2.0$, giving a frequency of the flow equal to $\omega=1 \mathrm{rad} / \mathrm{s}$ and $\omega=2 \mathrm{rad} / \mathrm{s}$ respectively. The gusts can be seen to be purely vortical, meaning that they are divergence free and convected with the mean flow $\left(\mathbf{a} \cdot \mathbf{k}=0\right.$ and $\left.k_{1} U_{\infty}=\omega\right)$. The vortical disturbances are added to the flow at the domain boundaries in three stages. First, the gusts are subtracted from the solution in all boundary cells using Eq. (21). A characteristic analysis can then be performed based only on flow induced disturbances. After the ghost cells have been set up to enable outgoing characteristics to exit the domain, the gusts are finally added back to all boundary and ghost cells. The physical time used in Eq. (21) is set according to the Harmonic Balance time level.

To achieve a minimum of 23 points per gust wavelength, the far field boundaries were placed at different distances from the airfoil for each reduced frequency while the cell count was kept approximately the same. A summary of the domain dimensions is given in table 1. These values compare well with numbers reported by other authors on this benchmark problem ${ }^{21,22}$

Table 1: Size of domain presented in figure 1

\begin{tabular}{cccc}
\hline$k$ & $d_{1}$ & $d_{2}$ & $d_{3}$ \\
1.0 & $15 c$ & $20 c$ & $25 c$ \\
2.0 & $10 c$ & $20 c$ & $25 c$ \\
\hline
\end{tabular}

\section{II.D.2. Cascade Gust Interaction}

The second benchmark problem was defined by Envia in Ref. 17 to study tone noise generated when rotating fan wakes interact with a two dimensional OGV cascade. This creates the well known Tyler-Sofrin spinning modes ${ }^{23}$ which in a two dimensional cascade are given as follows

$$
k_{m}=n k_{N_{1}}+l k_{N_{2}}, \quad l=\ldots,-1,0,1, \ldots
$$

Here, $k_{m}$ is the tangential wave number of the spinning mode and $n$ the harmonic number of the incoming rotor wake. The tangential wave numbers $k_{N_{1}}$ and $k_{N_{2}}$ are calculated as $2 \pi$ divided by the spacing of the rotor and stator blades respectively. Vortical velocity gusts containing three harmonics $(n=3)$ were specified at the inlet of the computational domain, which is schematically depicted in figure 3

A structured grid with a C-grid topology around the airfoil was employed. Referring to Eq. (23), the grid resolution was chosen to give approximately 30 points per wavelength in the tangential direction for the 


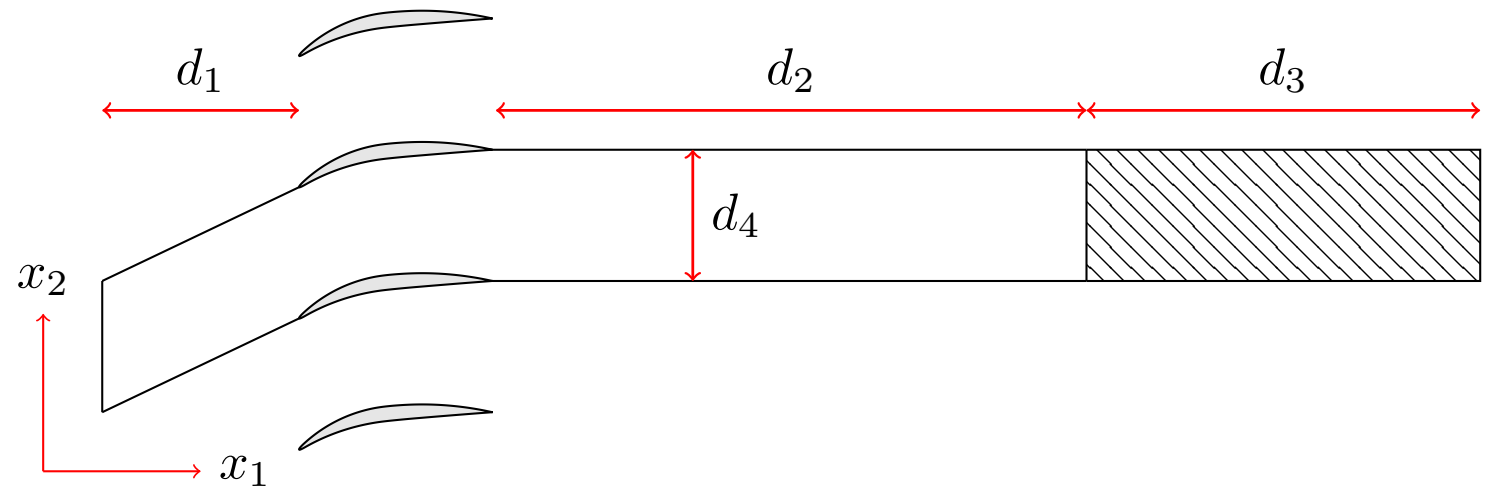

Figure 3: Computational domain for Cascade Gust Interaction problem.

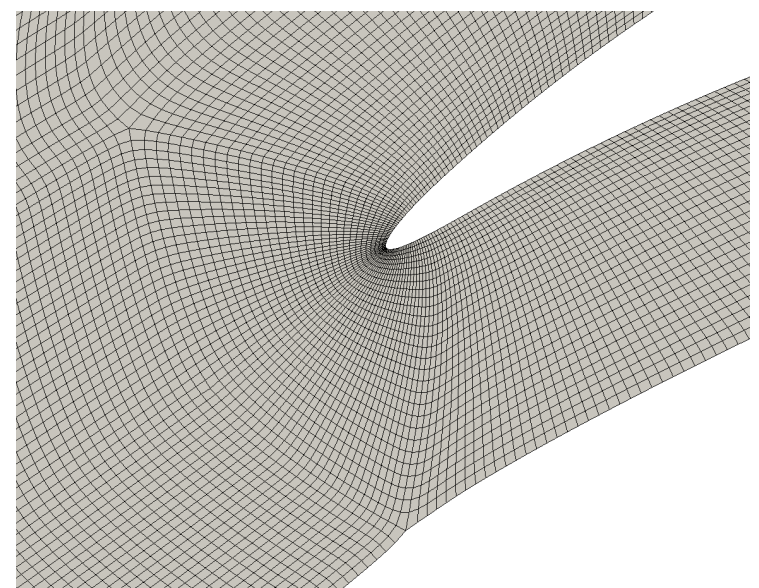

(a) Leading edge.

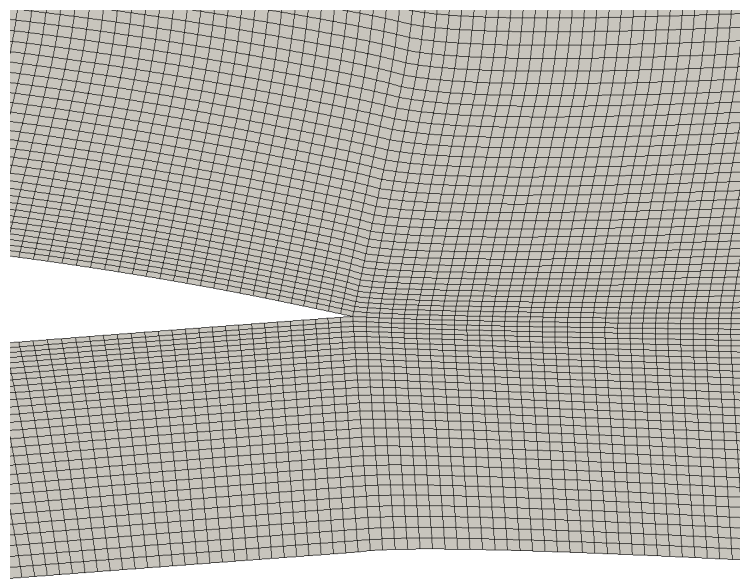

(b) Trailing edge.

Figure 4: Mesh for Outlet Guide Vane (OGV). 
interaction mode corresponding to $n=3$ and $l=2$. Resolution of lower harmonic interaction modes and the rotor wakes were higher. A detailed view of the mesh is presented in figure 4.

A buffer zone was applied in this case as well to cancel out any disturbances before they reached the outlet. Damping is performed on the conservative variables by adding a source term to the right hand side of Eq. (10)

$$
\ldots=-\varepsilon\left(x_{1}\right)\left(\mathcal{Q}^{*}-\overline{\mathcal{Q}^{*}}\right)
$$

Overline is used to denote time averaging and $\varepsilon\left(x_{1}\right)$ is a scaling factor. It increases smoothly from 0 at the beginning of the buffer zone to a maximum value of $10 / \mathcal{T}$ at the outlet, where $\mathcal{T}$ is the residence time of a convected disturbance inside the buffer zone. The time average can be obtained analytically as an algebraic average of the solution at each time level. The fluctuations can thus be calculated by means of a matrix multiplication

$$
\left(\mathcal{Q}^{*}-\overline{\mathcal{Q}^{*}}\right)=G \mathcal{Q}^{*}
$$

$G$ is here a matrix with the same structure as $D$, whose elements are given by

$$
g_{m, l}=\delta_{m, l}-\frac{1}{N_{t}}
$$

The damping can now simply be included by adding $\varepsilon\left(x_{1}\right) G$ to $D$ in the buffer zone. The size of the computational domain, including the buffer zone, is summarized in table 2.

Table 2: Size of domain presented in figure 3

\begin{tabular}{cccc}
\hline$d_{1}$ & $d_{2}$ & $d_{3}$ & $d_{4}$ \\
$1.5 c$ & $3 c$ & $2 c$ & $(2 / 3) c$ \\
\hline
\end{tabular}

A free slip boundary condition without explicit enforcement of the Kutta condition was set along the airfoil surface for this benchmark problem as well. Vortical disturbances were specified on top of the steady mean flow at the inlet using the one-dimensional absorbing formulation presented previously. The mean flow at the inlet was obtained from tangential averages of a steady state solution satisfying the following conditions $^{17}$

$$
\begin{aligned}
p_{0, \text { in }} & =101325 \mathrm{~Pa} \\
T_{0, \text { in }} & =288.333 \mathrm{~K} \\
\alpha_{\text {in }} & =36^{\circ}
\end{aligned} \quad p_{\text {out }}=0.92 P_{0, \text { in }}
$$

Subscript "0" denotes stagnation properties and $\alpha$ is the flow angle. This gave the values $\mathbf{U}_{\infty}=120.207 \mathbf{i}+$ $87.335 \mathrm{j} \mathrm{m} / \mathrm{s}, \rho_{\infty}=1.11109 \mathrm{~kg} / \mathrm{m}^{3}$ and $p_{\infty}=88440.2 \mathrm{~Pa}$. A buffer zone before the outlet enabled usage of a static pressure boundary condition for the acoustic analysis as well. The velocity gusts are furthermore defined as follows

$$
\begin{aligned}
\mathbf{u}^{\prime} & =\sum_{n=1}^{3} \epsilon_{n} a_{\infty} \mathbf{a} \cos (n(\mathbf{k} \cdot \mathbf{x}-\omega t)) \\
\mathbf{a} & =\frac{\sqrt{2}}{2}\left[\begin{array}{c}
1 \\
-1
\end{array}\right] \\
\mathbf{k} & =\left[\begin{array}{l}
k_{1} \\
k_{2}
\end{array}\right]
\end{aligned}
$$

Here, $a_{\infty}=\sqrt{\gamma R T_{0, \text { in }}}$ is the ambient speed of sound. The gust amplitudes and wave numbers are furthermore given by 


$$
\begin{aligned}
& \epsilon_{1}=5 \cdot 10^{-3}, \quad \epsilon_{2}=3 \cdot 10^{-3}, \quad \epsilon_{3}=7 \cdot 10^{-4} \\
& k_{1}=k_{2}=\frac{11 \pi}{9 c}
\end{aligned}
$$

The tangential wave number of the gusts corresponds to a fan stage with 11 fan blades and 27 outlet guide vanes. This information was used to set the phase shift according to Eq. (15). To ensure that the gusts were convected with the flow, i.e. remaied vortical, the frequency (or machine rpm) was set to satisfy $\mathbf{k} \cdot \mathbf{U}_{\infty}=\omega$. This gave an angular frequency of $\omega=796.90 \mathrm{rad} / \mathrm{s}$ for $c=1 \mathrm{~m}$, which is close to the reference value of $801.98 \mathrm{rad} / \mathrm{s}$ given in the problem description. ${ }^{17}$ An alternative approach could have been to adjust the inlet stagnation pressure in the steady state solution until the inlet velocity gives the frequency specified in the problem description.

\section{Results}

\section{III.A. Single Airfoil Gust Response}

Results for the first benchmark problem were obtained by running the Harmonic Balance solver with $N_{h}=2$ harmonics for all cases. Including higher harmonics was deemed unnecessary since the velocity gust only contains one harmonic with a small relative amplitude. This implies that the problem is close to linear and that $N_{h}=1$ would in fact suffice. The assumption was verified by checking the RMS pressure amplitude of the second harmonic along the airfoil surface for the loaded airfoil at $k=2.0$. It was found that the amplitude never exceeded $6.5 \%$ of the total RMS pressure amplitude, indicating that only small non-linear effects are present.

Contour plots of axial velocity and pressure fluctuations for the symmetric airfoil at $k=2.0$ are presented in figure $5 \mathrm{a}$ and $5 \mathrm{~b}$ respectively.

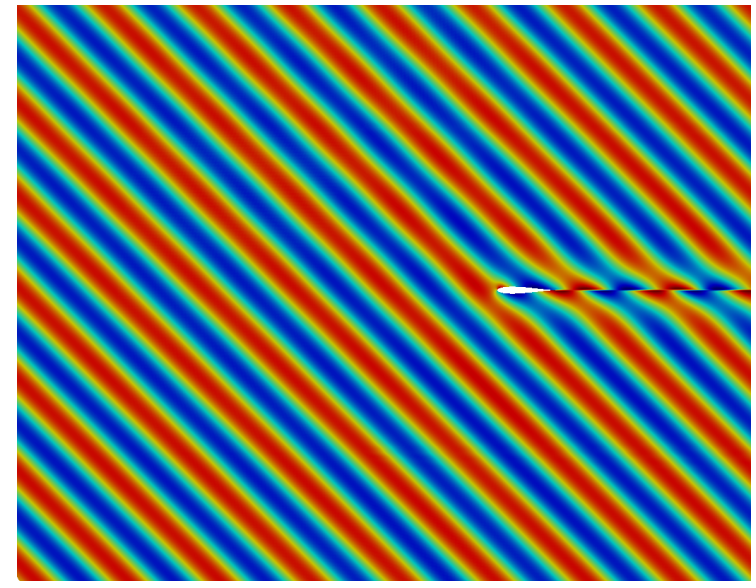

(a) Axial velocity gusts $\left(u_{x}^{\prime}\right)(\mathrm{m} / \mathrm{s}), k=2.0$.

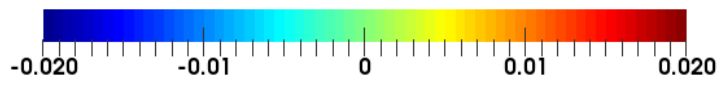

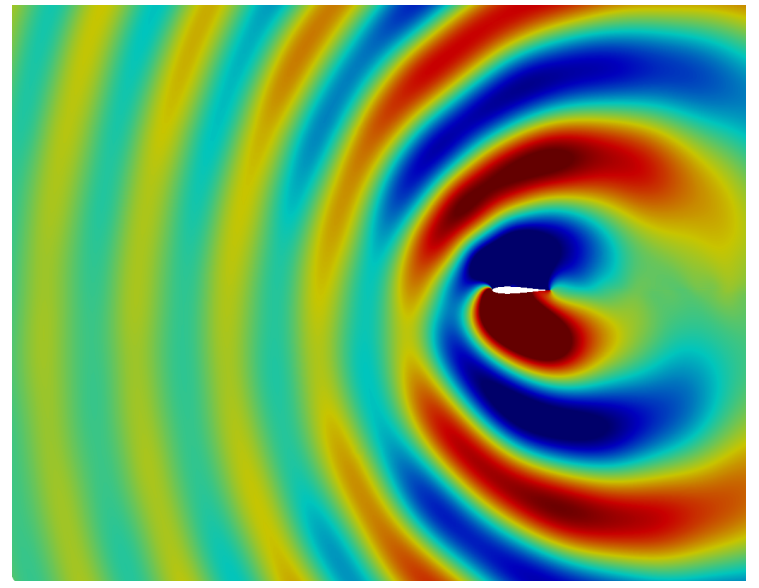

(b) Pressure response $\left(p^{\prime}\right)(\mathrm{Pa}), k=2.0$.

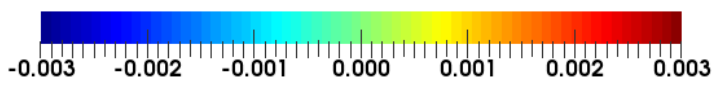

Figure 5: Contours of velocity and pressure fluctuations for symmetric airfoil, $k=2.0$.

The acoustic response is seen to have a dipole nature and radiate radially out from the airfoil surface. Results from G3D::Flow has also been compared to results submitted to the Fourth Computational Aeroacoustics Workshop on Benchmark Problems. ${ }^{24}$ For brevity the comparison was however limited to two codes, namely GUST3D and BASS. In addition, results from the BASS code published in Ref. 22 were used for the cambered airfoil at $k=2.0$ when results were missing in Ref. 24. A thorough review of all results contributed to the workshop, including those from GUST3D and BASS, has been made by Scott. ${ }^{21}$ This review will be used to quantify on the accuracy of the numbers presented hereinafter.

In figure 6 the RMS pressure as a function of axial chord at pressure and suction side of the airfoil is presented. All results have been normalized by $\rho_{\infty} U_{\infty}^{2}$. 


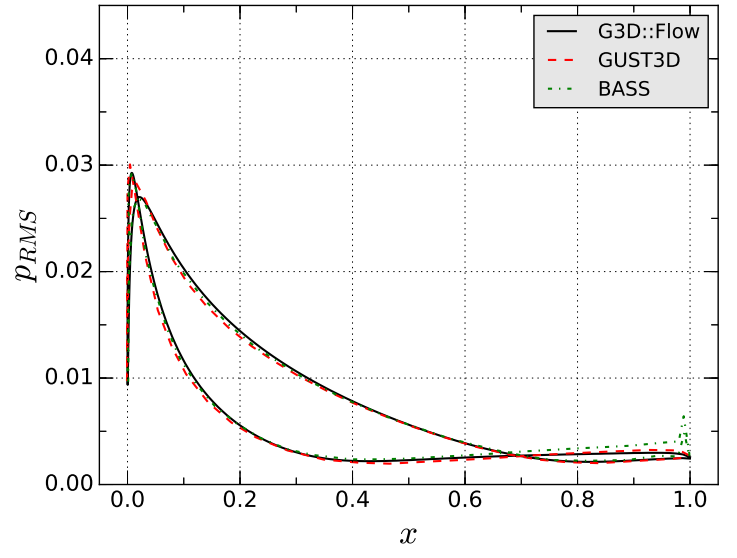

(a) Symmetric Airfoil, $k=1.0$.

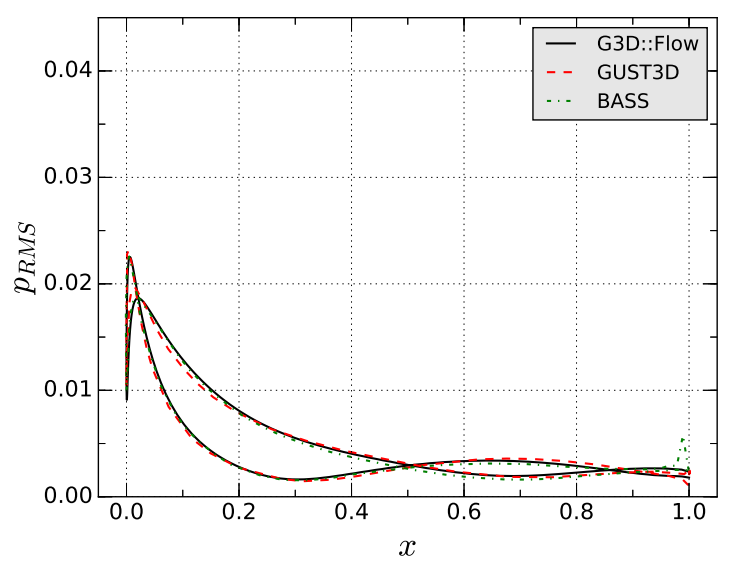

(c) Symmetric Airfoil, $k=2.0$.

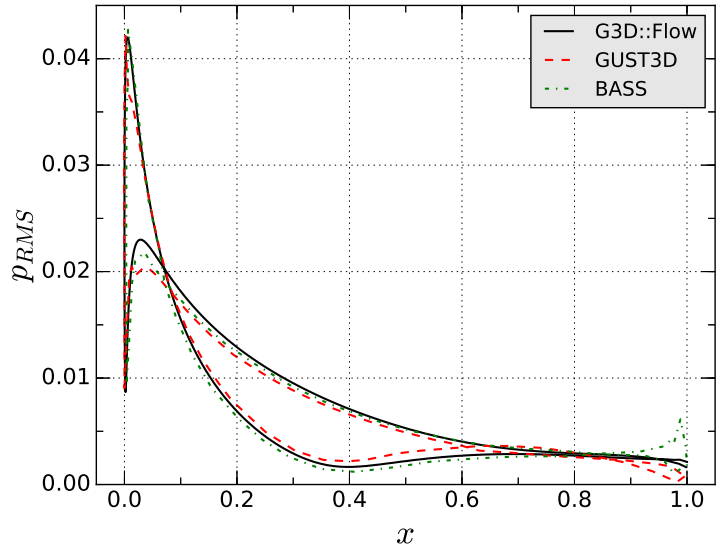

(b) Cambered Airfoil, $k=1.0$.

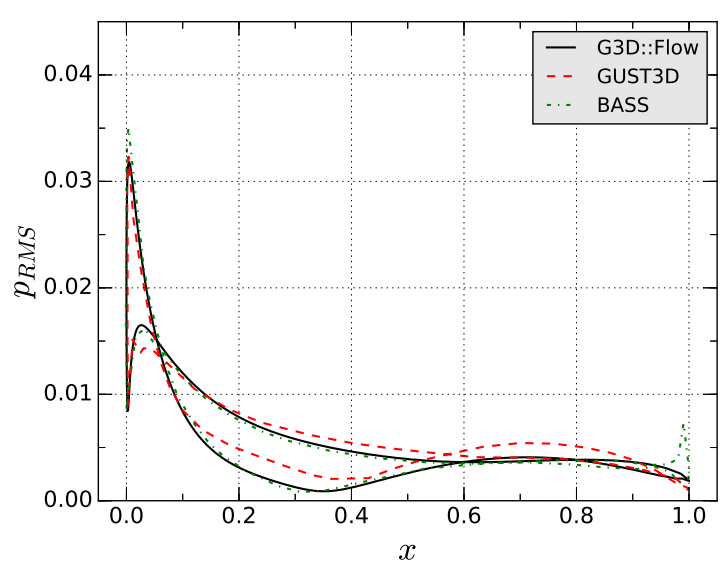

(d) Cambered Airfoil, $k=2.0$.

Figure 6: RMS pressure $\left(\overline{p^{2}}\right)^{0.5}$ on airfoil surface. 
For the symmetric airfoil the agreement between all codes is excellent at both reduced frequencies. Very good agreement is also observed for the cambered airfoil except at $k=2.0$, where the GUST3D code stands out slightly. It should however be noted that the GUST3D and BASS codes were found to be the best, i.e. most consistent, in this case by Scott. ${ }^{21}$ In summary the G3D::Flow Harmonic Balance solver is seen to produce a satisfactory pressure response at the airfoil surface.

To investigate the accuracy with which the solver propagates sound, the acoustic intensity was furthermore extracted from the solution on a circle of radius $1 c$. The results are presented in figure 7 .

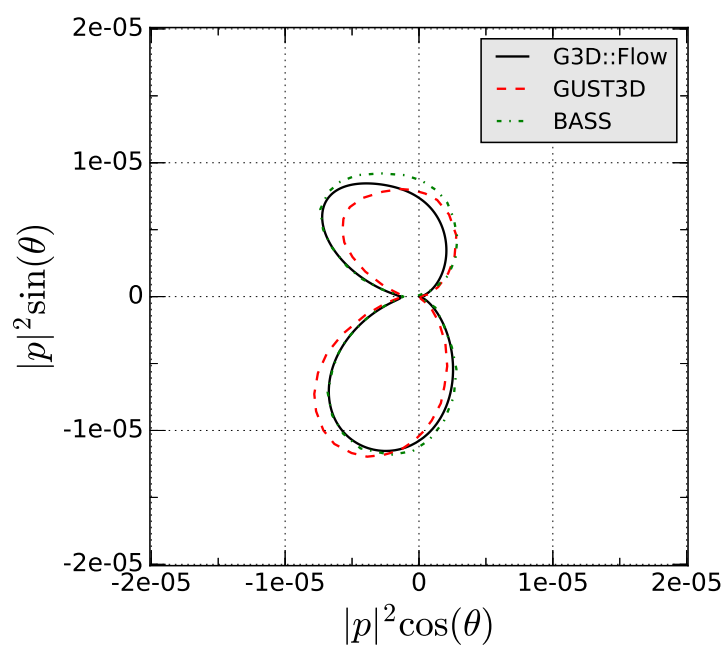

(a) Symmetric Airfoil, $k=1.0$.

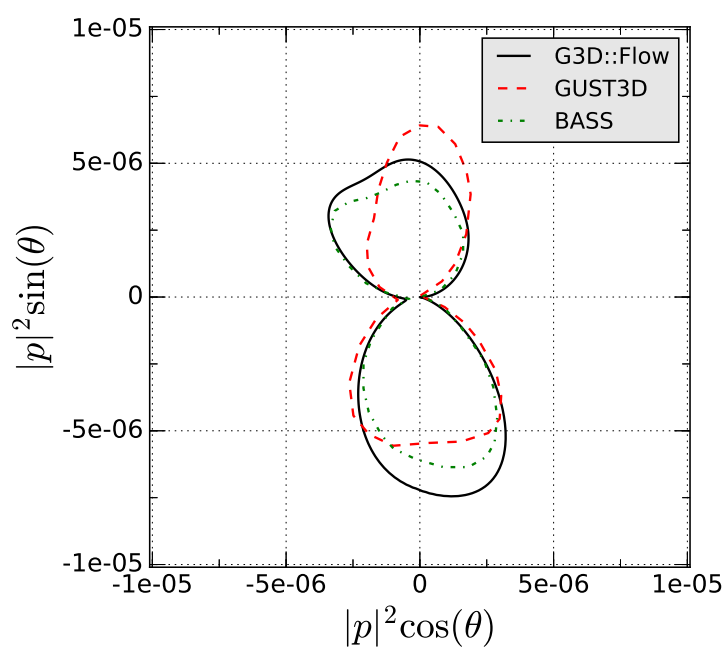

(c) Symmetric Airfoil, $k=2.0$.

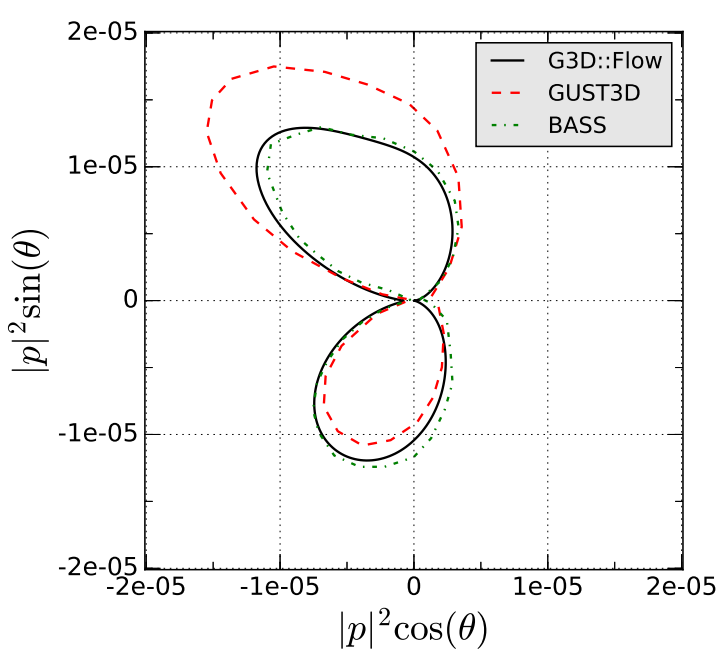

(b) Cambered Airfoil, $k=1.0$.

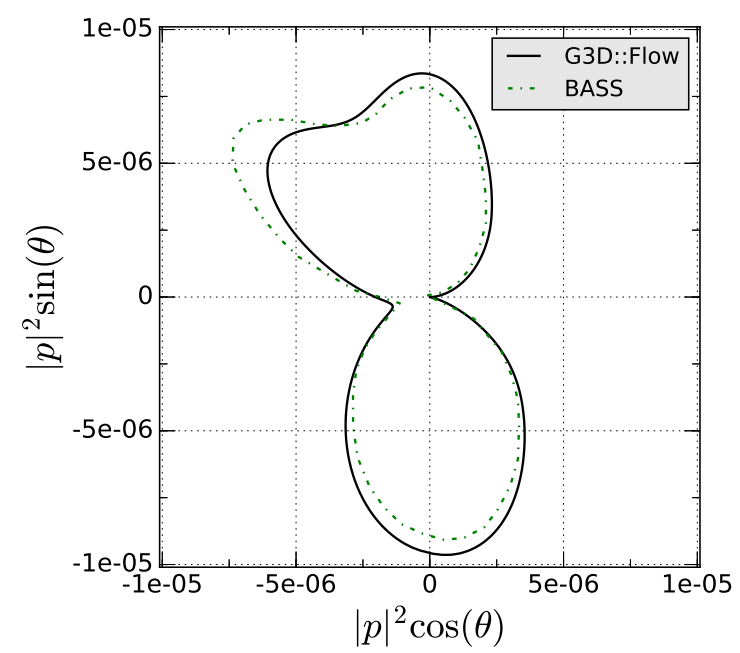

(d) Cambered Airfoil, $k=2.0$. Compared to Ref. 22

Figure 7: Acoustic intensity $\overline{p^{2}}$ on circle with $R=1 c$.

Results from all codes show good agreement, with the least discrepancy for the symmetric airfoil case at the lower reduced frequency. This is expected since the combination of an unloaded airfoil and lower reduced frequency should be the least challenging setup to predict. At the lower reduced frequency cases the BASS and GUST3D codes were considered to give the best results for the symmetric airfoil and the BASS code alone to be the best for the loaded airfoil. ${ }^{21}$ This shows that the G3D::Flow code does a good job in propagating the sound for these two cases. For the higher reduced frequency no best candidate was found by Scott. ${ }^{21}$ Good agreement is however observed between G3D::Flow and BASS for both airfoil geometries.

Acoustic radiation was further investigated by extracting the acoustic intensity on a circle of radius $4 c$ and the results are presented in figure 8 . 


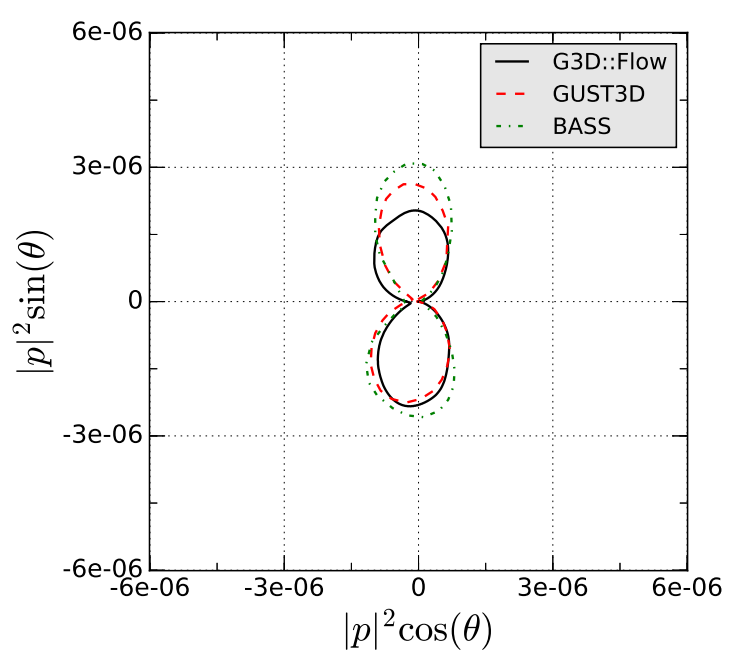

(a) Symmetric Airfoil, $k=1.0$.

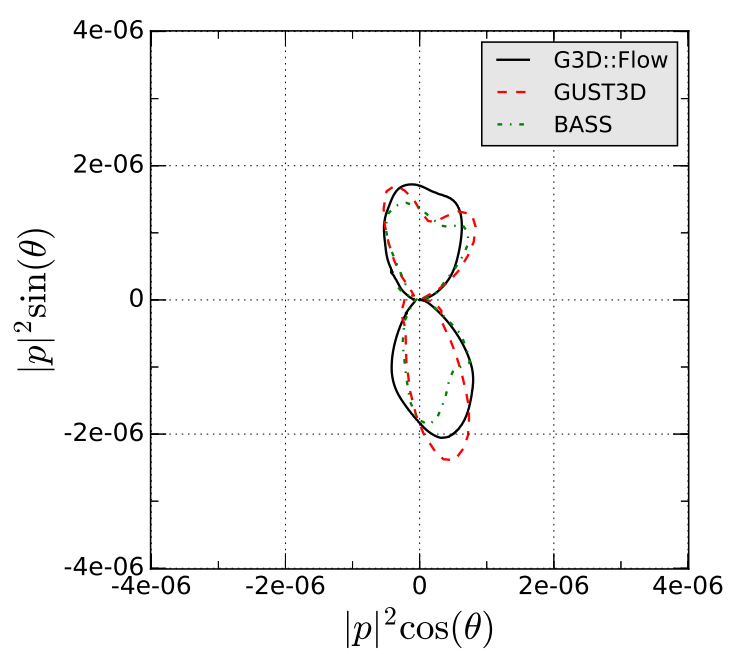

(c) Symmetric Airfoil, $k=2.0$.

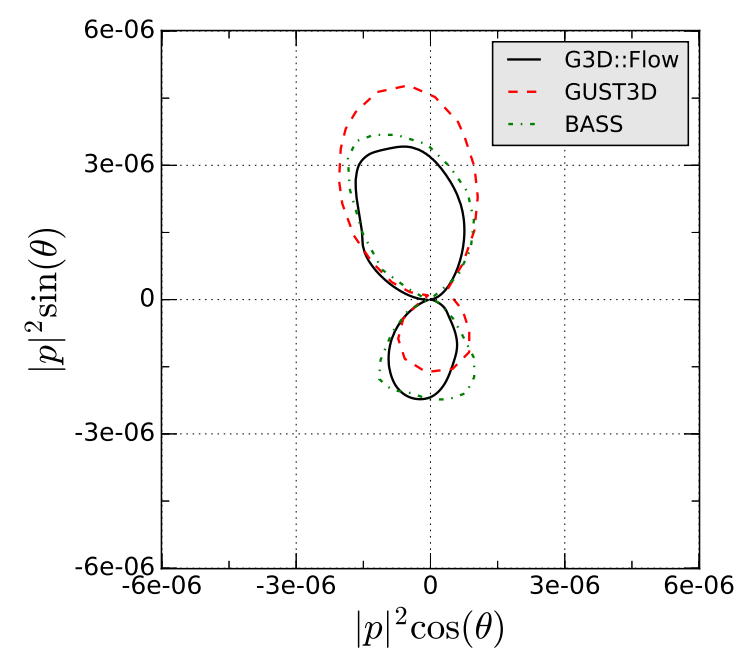

(b) Cambered Airfoil, $k=1.0$.

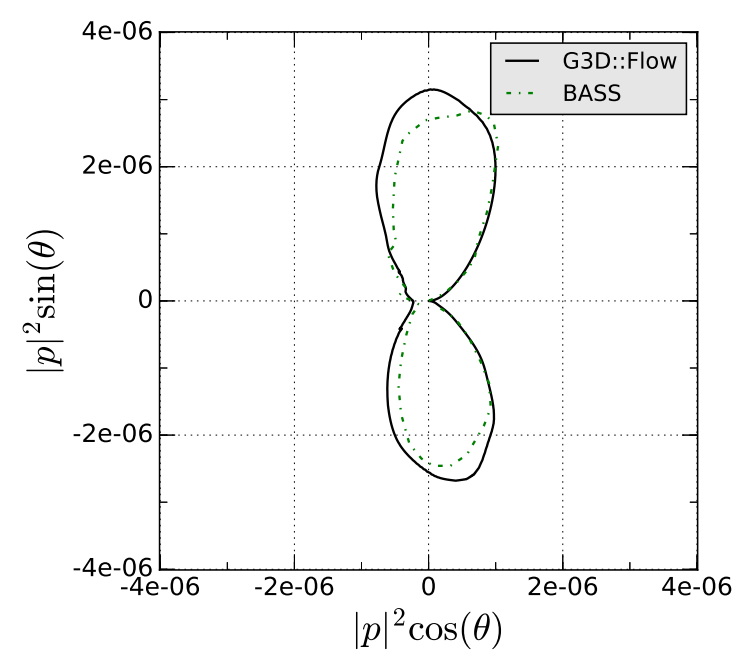

(d) Cambered Airfoil, $k=2.0$. Compared to Ref. 22

Figure 8: Acoustic intensity $\overline{p^{2}}$ on circle with $R=4 c$. 
As expected the discrepancy between the codes is the largest in this case, owing to the complexity of accurately propagating the acoustic signal over larger distances. At the lower reduced frequency the GUST3D and BASS codes were considered the best for the symmetric and cambered airfoil respectively. ${ }^{21}$ The agreement between G3D::Flow and each respective code for these two cases is satisfactory. For the symmetric airfoil at $k=2.0$ the G3D::Flow code is seen to not be able to reproduce the same type of directivity pattern as GUST3D and BASS. This may be due to insufficient mesh resolution in the G3D::Flow simulations, since the sampling circle cuts through the H-grid where a coarser mesh was employed. It must however be appreciated that similar to the smaller sampling circle, no best candidate exist for these results. ${ }^{21}$

\section{III.B. Cascade Gust Interaction}

The Harmonic Balance solver was run with $N_{h}=3$ harmonics to obtain results for the second benchmark problem. This represents the minimum harmonic content necessary to resolve the specified fan wake, and higher frequencies generated will not be resolved by the time spectral derivative. Initially the solver was run with the one dimensional absorbing formulation at both inlet and outlet. This was found to be insufficient since spurious reflections from especially the outlet quickly contaminated the solution. Converged results were obtained by implementing the buffer zone, in combination with a very strict CFL number constraint of 0.2 .

The discrete Fourier transform defined in Eq. (5) was used to obtain the amplitude of each individual harmonic in the solution. These were used to recreate the solution in the time domain, by evaluating Eq. (3) for each individual Fourier coefficient. An example of the axial velocity and pressure mode for $n=2$ is presented in figure 9 .

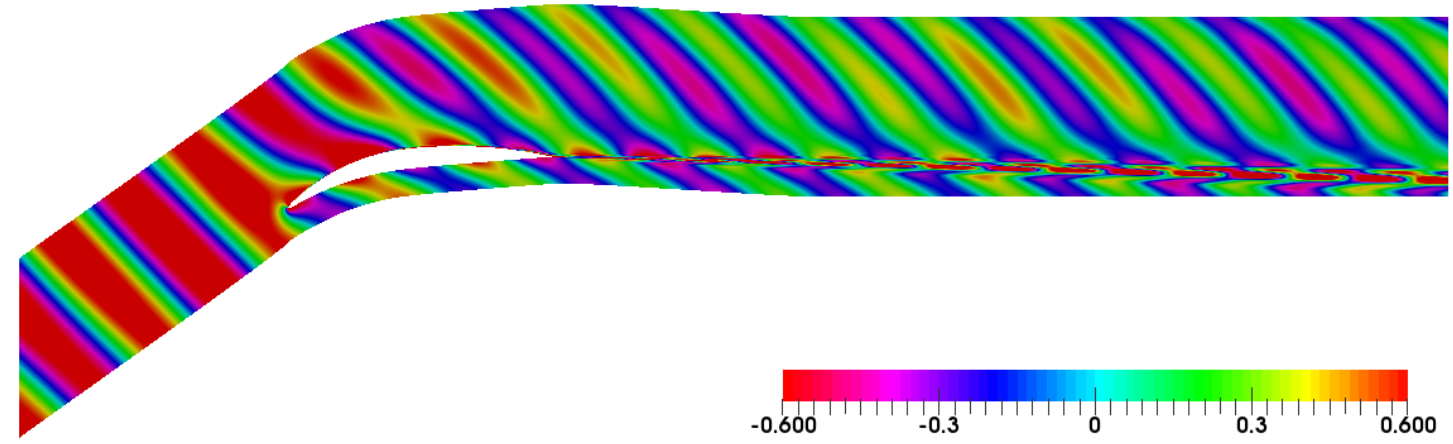

(a) Axial velocity fluctuations at frequency $2 \omega$

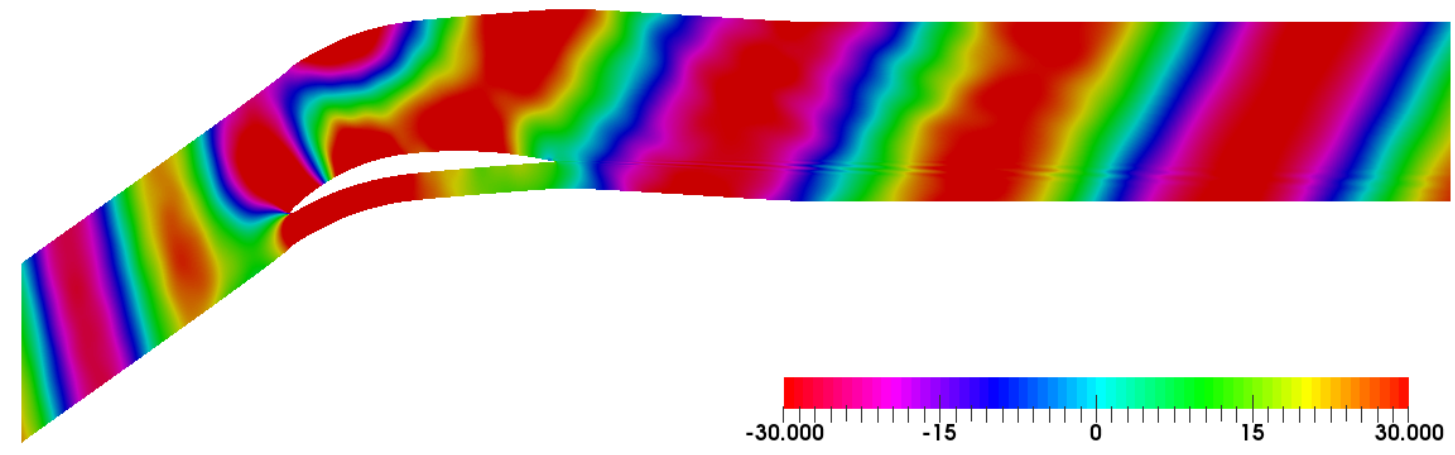

(b) Pressure fluctuations at frequency $2 \omega$

Figure 9: Contours of axial velocity and pressure fluctuations.

The second harmonic of the rotor wake is clearly visible upstream of the OGV vane. Vorticity is also seen to be shed from the trailing edge as caused by the transient lift that the airfoil experiences from the incoming rotor wakes. One upstream and one downstream running pressure mode can also be identified in 
figure $9 \mathrm{~b}$. The downstream running pressure mode can be seen to experience some wiggles. This indicates that reflections are generated in the buffer zone or at the outlet. The sound pressure level (SPL) of each individual harmonic was furthermore calculated along the airfoil pressure and suction side. They are compared to results published by Coupland ${ }^{25}$ using the HYDRA and HYDLIN codes in figure 10.

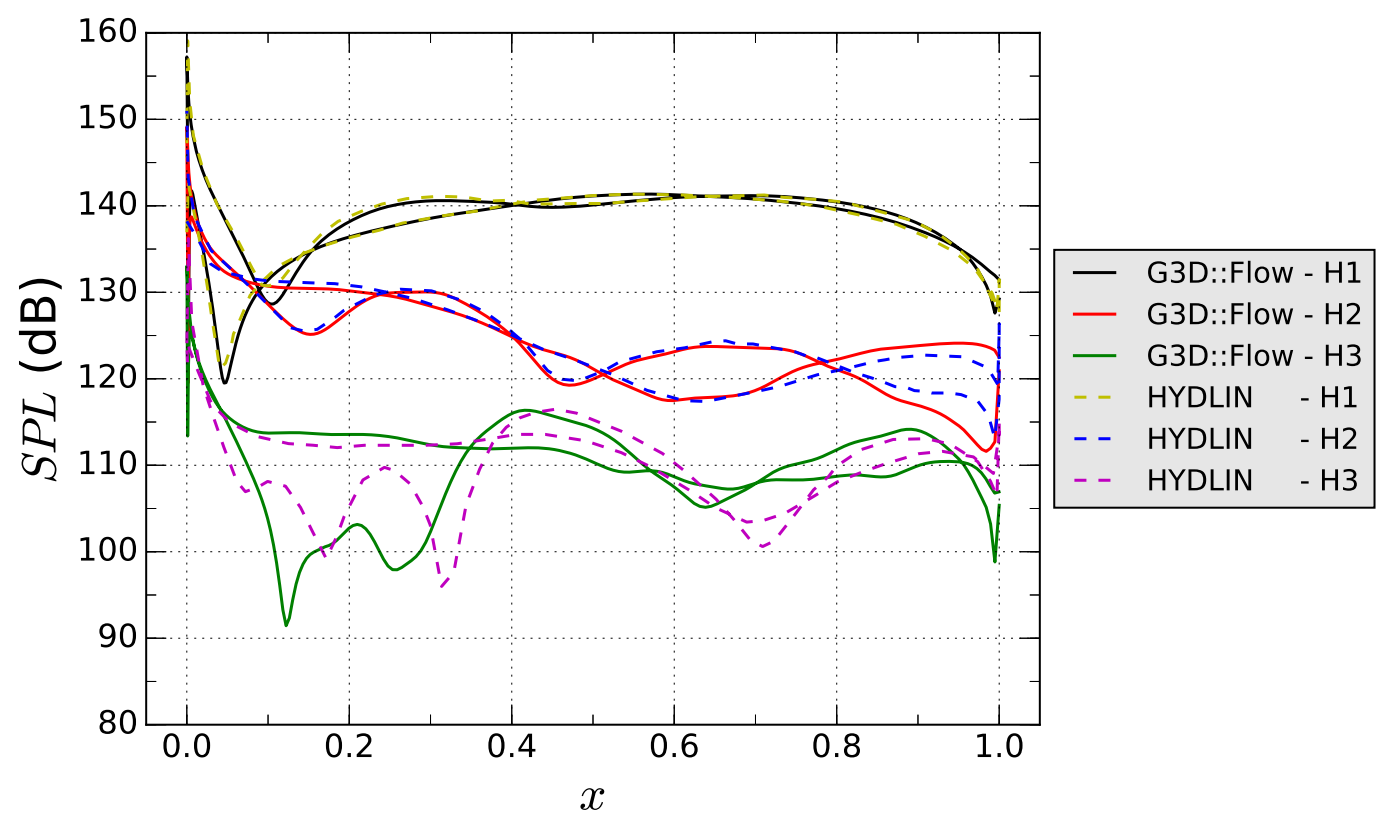

Figure 10: SPL on OGV surface for each individual harmonic.

Very good agreement is observed for the first and second harmonic, whereas notable discrepancies are observed for the third one. The discrepancies are taken as another indicator that reflections are indeed generated in the buffer zone or at the outlet. It is therefore believed that higher order absorbing boundary conditions could improve the results presented above. A longer buffer zone, possibly in combination with grid stretching, could be another way of minimizing reflections. It must however be appreciated that the method of damping the conservative variables employed here inevitably will generate a non-physical response in the solution. This stems from the fact that an even suppression of fluctuations in the conservative variables does not imply an even suppression of fluctuations in primitive variables, such as the pressure. Some of the reflections seen in the solution might thus be inevitable when using a buffer zone technique.

\section{Conclusions}

A Harmonic Balance method for turbomachinery tonal noise predictions has been presented and validated against two relevant problems from the Fourth Computational Aeroacoustics Workshop on Benchmark Problems hosted by NASA. Implementing the Harmonic Balance solver into an existing CFD solver using explicit time marching was found to be very convenient. Another strength of the method is that the flow field can be readily obtained at any arbitrary time instant along the time period. This makes the implementation of new features, such as phase-shifted periodic boundary conditions or the buffer zone a much simpler task compared to doing it in a standard time accurate solver.

Very good agreement with two aeroacoustics codes, GUST3D and BASS, was observed for the single airfoil gust response problem. The discrepancies seen were generally not larger than those reported between other codes by Scott. ${ }^{21}$ It was shown that only small non-linearities existed in the first benchmark problem by investigating RMS pressure amplitudes on the airfoil surface of the individual harmonics resolved in the Harmonic Balance computation. Considering that the gusts had a similar relative amplitude in the cascade gust interaction problem, non-linearities should play a small role here as well. Results presented in this paper therefore only verifies that the Harmonic Balance method accurately predicts individual frequencies of the flow. Coupling between different harmonics enabled by the non-linear formulation remains an open question that should be further investigated in the future. If these effects are more pronounced, frequencies beyond 
those resolved in the Harmonic Balance simulation can furthermore be generated if $N_{h}$ is chosen too small. In this event, it is likely also necessary to employ filtering similar to the method proposed by LaBryer et al. ${ }^{26}$ Otherwise, aliasing of higher generated frequencies onto the resolved ones will occur, generating inaccurate acoustic results.

For the cascade gust interaction problem the Harmonic Balance solver was found to be in very close comparison with the linear HYDLIN code for the lower harmonics. The discrepancies seen were furthermore thought to be due to insuficient absorbtion of outgiong waves at the boundaries in the G3D::Flow simulations. Convergence issues was also observed in the second benchmark problem which forced the use of a very strict CFL number of 0.2. It can therefore not be verified that the implicit treatment of the time spectral source term proposed by Campobasso et al. ${ }^{19}$ improves stability over an explicit treatment. Higher order absorbing boundary conditions and investigation into new solver technologies are therefore necessary.

\title{
Acknowledgments
}

This work is financially supported by the E.U. under the ULTIMATE ${ }^{\text {a }}$ Project funded by the European Commission within the Horizon 2020 Programme (2014-2020) under the Grant Agreement No 633436. The authors would also like to acknowledge the Swedish National Infrastructure for Computing (SNIC) for providing computer resources at the National Supercomputing Centre (NSC) in Linköping, Sweden.

\section{References}

\begin{abstract}
${ }^{1}$ Peake, N. and Parry, A. B., "Modern Challenges Facing Turbomachinery Aeroacoustics," Annual Review of Fluid Mechanics, Vol. 44, No. 1, January 2012, pp. 227-248.

${ }^{2}$ Sharma, A. and Chen, H., "Prediction of aerodynamic tonal noise from open rotors," Journal of Sound and Vibration, Vol. 332, No. 16, August 2013, pp. 3832-3845.

${ }^{3}$ Envia, E., Wilson, A. G., and Huff, D. L., "Fan Noise: A Challenge to CAA," International Journal of Computational Fluid Dynamics, Vol. 18, No. 6, August 2004, pp. 471-480.

${ }^{4}$ Parry, A. B., Kingan, M., and Tester, B. J., "Relative importance of open rotor tone and broadband noise sources," AIAA Paper 2011-2763, 17th AIAA/CEAS Aeroacoustics Conference, Portland, OR, 5-8 June 2011.

${ }^{5}$ Gerolymos, G. A., Michon, G. J., and Neubauer, J., "Analysis and Application of Chorochronic Periodicity in Turbomachinery Rotor/Stator Interaction Computations," Journal of Propulsion and Power, Vol. 18, No. 6, November-December 2002, pp. 1139-1152.

${ }^{6}$ Olausson, M., Turbomachinery Aeroacoustic Calculations using Nonlinear Methods, Ph.D. thesis, Division of Fluid Dynamics, Department of Applied Mechanics, Chalmers University of Technology, Gothenburg, 2011.

${ }^{7}$ Colin, Y., Blanc, F., Caruelle, B., Barrois, F., and Djordjevic, N., "Computational stratedy for predicting CROR noise at low-speed Part II: Investigation of the Noise Sources Computation with the Chorochronic Approach," AIAA Paper 2012-2222, 18th AIAA/CEAS Aeroacoustics Conference, Colorado Springs, CO, 4-6 June 2012.

${ }^{8} \mathrm{He}$, L. and Ning, W., "Efficient Approach for Analysis of Unsteady Viscous Flows in Turbomachines," AIAA Journal, Vol. 36, No. 11, November 1998, pp. 2005-2012.

${ }^{9}$ Hall, K. C., Ekici, K., Thomas, J. P., and Dowell, E. H., "Harmonic Balance Methods Applied to Computational Fluid Dynamics Problems," International Journal of Computational Fluid Dynamics, Vol. 27, No. 2, January 2013, pp. 52-67.

${ }^{10}$ Hall, K. C., Jeffrey, P. T., and Clark, W. S., "Computation of Unsteady Nonlinear Flows in Cascades Using a Harmonic Balance Technique," AIAA Journal, Vol. 40, No. 5, May 2002, pp. 879-886.

${ }^{11}$ McMullen, M. S. and Jameson, A., "The Computational Efficiency of Non-Linear Frequency Domain Methods," Journal of Computational Physics, Vol. 212, No. 2, March 2006, pp. 637-661.

${ }^{12}$ Van Zante, D. E. and Envia, E., "Prediction of the Aero-Acoustic Performance of Open Rotors," GT2014-26413, ASME Turbo Expo 2014, Düsseldorf, Germany, 16-20 June 2014.

${ }^{13}$ Frey, C., Ashcroft, G., and Kersen, H.-P., "Simulations of Unsteady Blade Row Interactions Using Linear and Non-Linear Frequency Domain Methods," GT2015-43453, ASME Turbo Expo 2015, Montréal, Canada, 15-19 June 2015.

${ }^{14}$ Pinelli, L. et al., "On the Numerical Evaluation of Tone Noise Emissions Generated by a Turbine Stage: An In-Depth Comparison Among Different Computational Methods," GT2015-42376, ASME Turbo Expo 2015, Montréal, Canada, 15-19 June 2015.

${ }^{15}$ Hoffner, P.-A., Deconinck, T., Hirsch, C., Pascal, S., Ortun, B., Canard-Caruana, S., Rahier, G., and Caruelle, B., "Aeroacoustic Computations of Contra-Rotating Open Rotors using the Nonlinear Harmonic Method and a Chorochronic Approach," GT2012-68982, ASME Turbo Expo 2012, Copenhagen, Denmark, 11-15 June 2012.

${ }^{16}$ Scott, J. R., "Single Airfoil Gust Response Problem," Fourth Computational Aeroacoustics (CAA) Workshop on Benchmark Problems, edited by M. D. Dahl, NASA/CP-2004-212954, Brook Park, OH, 20-22 October 2003, pp. 45-58.

${ }^{17}$ Envia, E., "Cascade-Gust Interaction," Fourth Computational Aeroacoustics (CAA) Workshop on Benchmark Problems, edited by M. D. Dahl, NASA/CP-2004-212954, Brook Park, OH, 20-22 October 2003, pp. 59-66.
\end{abstract}

${ }^{\mathrm{a}}$ Ultra Low emission Technology Innovations for Mid-century Aircraft Turbine Engines 
${ }^{18}$ Gopinath, A. K. and Jameson, A., "Application of the Time Spectral Method to Periodic Unsteady Vortex Shedding," AIAA Paper 2006-0449, 44th AIAA Aerospace Sciences Meeting and Exhibit, Reno, NV, 9-12 January 2006.

${ }^{19}$ Campobasso, M. S. and Baba-Ahmadi, M. H., "Analysis of Unsteady Flows Past Horizontal Axis Wind Turbine Airfoils Based on Harmonic Balance Compressible Navier-Stokes Equations With Low-Speed Preconditioning," Journal of Turbomachinery, Vol. 134, No. 6, November 2012, pp. 061020-1-011001-13.

${ }^{20}$ Sicot, F., Dufour, G., and Gourdain, N., "A Time-Domain Harmonic Balance Method for Rotor/Stator Interactions," Journal of Turbomachinery, Vol. 134, No. 1, January 2012, pp. 011001-1-011001-13.

${ }^{21}$ Scott, J., "Benchmark Solutions for Computational Aeroacoustics (CAA) Code Validation," IMECE2004-59865, ASME 2004 International Mechanical Engineering Congress and Exposition, Anaheim, CA, 13-19 November 2004.

${ }^{22}$ Hixon, R., Scott, J., Sawyer, S., and Nallasamy, M., "Application of a Nonlinear Computational Aeroacoustics Code to the Gust-Airfoil Problem," AIAA Journal, Vol. 44, No. 2, February 2006, pp. 323-328.

${ }^{23}$ Tyler, J. M. and Sofrin, T. G., "Axial Flow Compressor Noise Studies," SAE Technical Paper 620532, 1962, pp. 309-332.

${ }^{24}$ Scott, J. R., "Comparison of Solutions," Fourth Computational Aeroacoustics (CAA) Workshop on Benchmark Problems, edited by M. D. Dahl, NASA/CP-2004-212954, Brook Park, OH, 20-22 October 2003, pp. 461-479.

${ }^{25}$ Coupland, J., "Linear Unsteady CFD Analysis of the Cascade-Gust Interaction Problem," Fourth Computational Aeroacoustics (CAA) Workshop on Benchmark Problems, edited by M. D. Dahl, NASA/CP-2004-212954, Brook Park, OH, 20-22 October 2003, pp. 97-105.

${ }^{26}$ LaBryer, A. and Attar, P., "High Dimensional Harmonic Balance Dealiasing Techniques for a Duffling Oscillator," Journal of Sound and Vibration, Vol. 324, No. 3-5, 2009, pp. 1016-1038. 